А. В. Ивашко, А. А. Зуев

Национальный технический университет «ХПИ», Харьков, Украина

\title{
РАЗРАБОТКА МЕТОДОВ УДАЛЕНИЯ ДЕФЕКТОВ НА ИЗОБРАЖЕНИЯХ ЭНЕРГООБЪЕКТОВ, ПОЛУЧЕННЫХ В ПРОЦЕССЕ ДИСТАНЦИОННОГО МОНИТОРИНГА С ПОМОЩЬЮ БПЛА
}

\begin{abstract}
Целью статьи является разработка и экспериментальное исследование методов удаления дефектов на изображениях, полученных в результате дистанционного мониторинга энергообъектов с помощью БПЛА, вызванного построчным переносом изображения с матрицы камеры в устройство хранения. При проведении исследований использовались методы корреляционного оценивания относительного сдвига последовательностей, методы цифровой нелинейной фильтрации, математическое моделирование в пакете Scilab. Предложены и программно реализованы методы подавления артефактов вызванных эффектом построчного переноса, не требующие анализа последовательности кадров и позволяющие устранять искажения, вызванные как наклоном камеры, так и ее вибрацией. Использование предложенных методов позволяет создать программное обеспечение для бортового компьютера БПЛА, которое может устранять артефакты на изображениях, полученных в процессе мониторинга в реальном масштабе времени. Получены расчетные соотношения, позволяющие определить предполагаемые значения сдвига строк изображения, вызванного эффектом построчного переноса, предложены и программно реализованы методы удаления искажений на изображениях энергообъектов, получаемых в процессе дистанционного мониторинга с помощью БПЛА.
\end{abstract}

Ключевые слов а: воздушные линии электропередачи, дистанционный мониторинг, БПЛА, построчный перенос, обработка изображений, медианная фильтрация, взаимнокорреляционная функция.

\section{Введение}

В последние годы, в развитых странах имеющих электроэнергетическую инфраструктуру значительной протяженности, таких как США, ЕС, Китай, Бразилия, РФ, проводятся мероприятия по дистанционному мониторингу сетей ЛЭП [1]. Уровень развития науки и техники, позволяет использовать для решения таких задач, помимо классических, принципиально новые методы. Среди которых следует отметить дистанционный мониторинг энергетической инфраструктуры с помощью беспилотных летающих аппаратов (БПЛА) [2-3], основанный на визуальном, тепловизионном и лазерном сканировании ЛЭП, с последующей обработкой полученных данных. Преимуществом такого метода мониторинга является возможность широкой автоматизации и комплексного контроля параметров ЛЭП, характеризующих как внешнее состояние объектов энергосистемы, так и ее основных электрических параметров.

Основным источником данных в процессе мониторинга являются различные фото- и видео- камеры, установленные на БПЛА. Это могут быть как камеры регистрирующие изображения в видимом диапазоне, так и различные виды тепловизионных и мультиспектральных камер. Серьезной проблемой, возникающей при фото и видеосъемке с применением матриц CMOS (КМOП-сенсор) и других типов матриц, использующих не моментальную фиксацию изображения, а построчное считывание, является так называемый эффект "роллинг-шаттер" или построчный перенос (англ. rolling shutter, раскатывающийся затвор). Поскольку матрица фиксирует изображение построчно, сверху вниз, то при движении объекта или камеры на обработку подается лишь информация с одной или нескольких строк. За время съемки кадра объект успевает сменить место- положение, что обусловлено характером движения БПЛА, и в результате участки одного кадра отображают разные моменты времени.

Такое запаздывание получения данных с матрицы относительно движения в кадре приводит к появлению хорошо заметных геометрических искажений объектов. Такие камеры имеют стоимость в несколько раз меньше, чем камеры, фиксирующие все изображение одновременно, таким образом, их применение позволяет существенно снизить стоимость системы мониторинга, но требует разработки специальных методов обработки полученных изображений, которые бы минимизировали геометрические искажения.

Пример изображения с артефактами построчного переноса приведен на рис. 1.

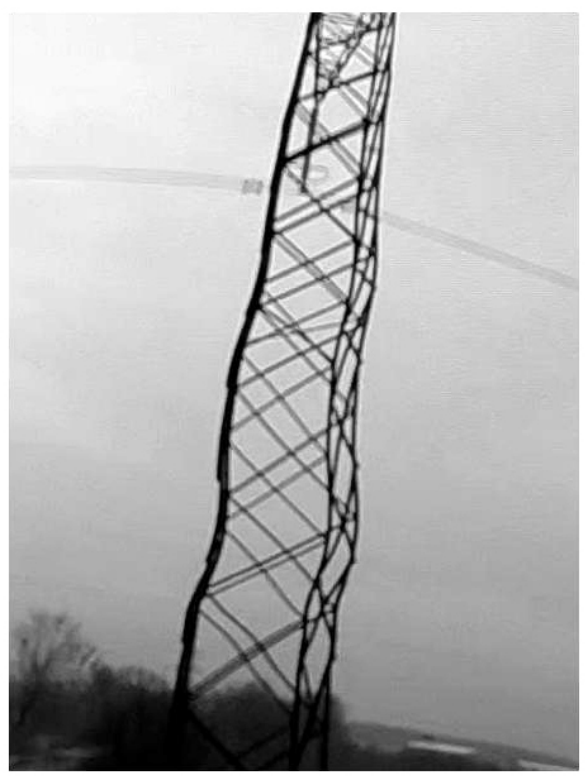

Рис. 1. Изображение опоры ЛЭП с артефактами построчного переноса 
На изображении хорошо видны волнообразные искривления, вызванные вибрацией размещенной на БПЛА камеры. Этот эффект может проявляться в виде наклона или искривления отдельных объектов или всего кадра, появления горизонтальных пересеченных полос, а иногда даже в хаотичном искажении кадра и расплывании изображения, так называемый "эффект желе".

Такие искажения существенно усложняют автоматическое выделение контуров и объектов на изображении, а также определение количественных и качественных характеристик объектов по результатам фото- и видеосъемки. Это, в свою очередь, приводит к снижению эффективности или невозможности применения автоматических методов обработки данных полученных в результате мониторинга.

\section{Анализ литературных данных и постановка проблемы}

В работе [4] решается задача оценки качества электрических сетей на основе видеосъемок уличных осветительных ламп. Авторы отмечают, что артефакты, связанные с эффектом построчного переноса затрудняют решение таких задач, и они должны быть удалены.

В последнее время предпринимался ряд попыток разработки алгоритма подавления артефактов, возникающих при построчном переносе. Которые, однако, не всегда обеспечивают требуемый результат. В [5] предлагается анализировать пары последовательных кадров в видеопотоке и вычислять предполагаемый вектор движения при помощи дифференциального локального алгоритма Лукаса-Канаде, а затем корректировать искаженные кадры при помощи так называемого целочисленного Р-преобразования. При разработке алгоритма предполагается, что камера движется только прямолинейно. Это требование, а также ограничение на степень размытия и отсутствие локальных движений в кадре, значительно ограничивает круг решаемых задач при помощи предлагаемого алгоритма. Например, задачи мониторинга энергообъектов, для которых, требуется движение по сложным, непрямолинейным траекториям. Также особенности движения малоразмерных БПЛА при воздействии приземных воздушных потоков делают применение такого алгоритма невозможным.

Несколько более реалистичная модель движения камеры представлена в [6]. Авторы учитывают колебания камеры и объекта и компенсируют артефакты построчного переноса с учетом не только прямолинейного, но и колебательного движения. Следует, однако, отметить, что представленные авторами алгоритмы автокалибровки и оценки частоты колебаний требуют значительных вычислительных затрат и не всегда применимы в случае встроенной микроконтроллерной реализации бортового компьютера БПЛА. В работах [7, 8] также анализируется последовательность кадров, и предпринимаются попытки выделения областей изображения, подверженных, артефактам связанных с эффектом построчного переноса. Приведенные алгоритмы тестировались на реальных видеопотоках, в том числе и полученных с БПЛА.

Общим недостатком рассмотренных методов является необходимость анализа нескольких последовательных кадров, что не всегда удобно при съемке в экстремальных условиях и требует повышенных вычислительных затрат. Кроме того, большинство известных алгоритмов поворачивают на фиксированный угол весь кадр и неудовлетворительно борются с дрожаниями.

Целью статьи является разработка и экспериментальное исследование методов удаления дефектов на изображениях, полученных в результате дистанционного мониторинга объектов энергетической инфраструктуры с помощью БПЛА, вызванного построчным переносом изображения с матрицы камеры в устройство хранения.

\section{Метод удаления артефактов изображений, связанных с эффректом построчного переноса}

Изображение размером $\mathrm{N}_{1} \times \mathrm{N}_{2}\left(\mathrm{~N}_{1}-\right.$ число строк, $\mathrm{N}_{2}$ - число столбцов) просматривается построчно и для каждой строки определяется предполагаемый горизонтальный сдвиг относительно предыдущей, вызванный перемещением камеры. Для этого вычисляется оценка взаимной корреляции двух смежных строк изображения

$$
\begin{gathered}
R_{k, k+1}(s)=\frac{1}{N_{2}} \sum_{i=1}^{N_{2}} a_{k, i} \cdot a_{k+1, i+s}, \\
-s_{\max } \leq s \leq s_{\max }
\end{gathered}
$$

где $\mathrm{a}_{\mathrm{k}, \mathrm{i}}$ - k-й элемент i-й строки изображения; s относительный сдвиг смежных строк, $\mathrm{s}_{\max }$ - предполагаемое максимальное значение сдвига.

Затем определяется значение относительного сдвига k-й и k+1-й строк изображения $\mathrm{s}_{\mathrm{k}}$, соответствующее максимальному значению взаимнокорреляционной функции (ВКФ) в диапазоне $\pm \mathrm{S}_{\max }$.

В случае, если ожидаемое значение относительного сдвига составляет менее одного пикселя, оно может быть определено как взвешенная оценка сдвига с учетом значений ВКФ:

$$
s_{k}=\sum_{s=-s_{\max }}^{s_{\max }} s \cdot R_{k, k+1}(s) / \sum_{s=-s_{\max }}^{s_{\max }} R_{k, k+1}(s) .
$$

Предполагаемый сдвиг каждой из строк изображения относительно первой строки может быть получен путем накопления сдвигов пар смежных строк

$$
s_{k}^{\prime}=\sum_{j=1}^{k} s_{k}
$$

Если значение текущего сдвига, вычисленное по формуле (3), оказывается дробным, оно должно быть округлено до ближайшего целого значения.

Другой подход к определению величины сдвига, вызванного перемещением камеры, в случае, ес- 
ли это значение составляет менее одного пикселя, состоит в том, что определяется сдвиг между строками, которые отстоят друг от друга на некоторое значение $\Delta \mathrm{k}$, большее единицы:

$$
R_{k, k+\Delta k}(s)=\frac{1}{N_{2}} \sum_{i=1}^{N_{2}} a_{k, i} \cdot a_{k+\Delta k, i+s} .
$$

После этого вычисляется значение максимума ВКФ в пределах $\pm \mathrm{s}_{\max }$ и соответствующий этому значению сдвиг $\mathrm{s}_{\mathrm{k}}$, и производится накопление вычисленных локальных сдвигов.

После определения сдвига каждой из строк изображения, вызванного перемещением камеры, этот сдвиг может быть скомпенсирован путем смещения строки на соответствующее число пикселей в противоположном направлении.

\section{Экспериментальное исследование предложенного метода}

Предлагаемые методы были реализованы в среде моделирования Scila. Они были экспериментально проверены на реальных изображениях объектов энергетической инфраструктуры, которые получены с установленной на БПЛА видеокамеры и подверженной воздействию эффекта построчного переноса. На рис. 2 изображен график зависимости величины сдвига строки в пикселях от ее номера для метода взвешенного оценивания (2) при $s_{\max }=5$.

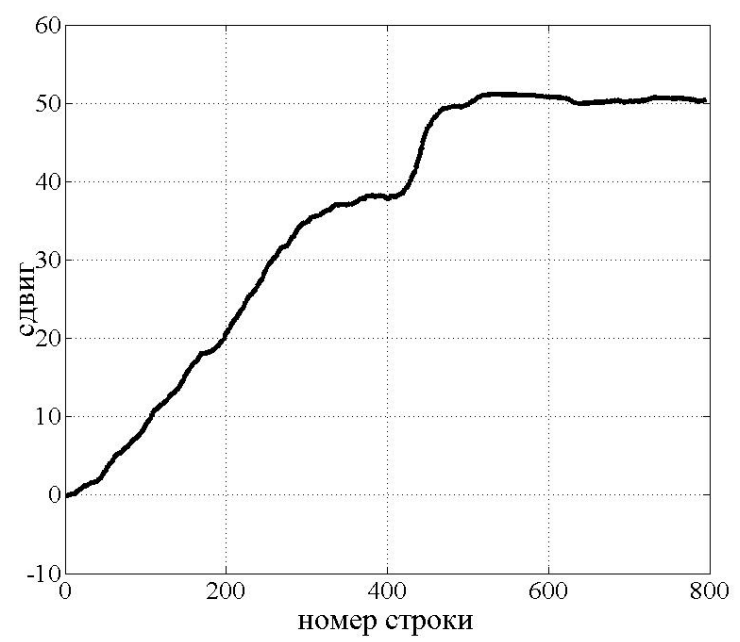

Рис. 2. График зависимости накопленного сдвига от номера строки для метода взвешенного оценивания для $\mathrm{s}_{\max }=5$

На рис. 3 приведены результаты обработки изображения, пораженного артефактами, связанными с эффектом построчного переноса. Применен алгоритм взвешенного оценивания с параметром $\mathrm{s}_{\max }=5$. В результате обработки снижен вызванный движением камеры наклон объекта и уменьшена амплитуда колебаний изображения объекта, хотя и несколько повышена их частота.

На рис. 4 изображен график зависимости сдвига строки от ее номера для метода максимума ВКФ при $\Delta \mathrm{k}=5$. На графике хорошо заметны дрожания, вызванные неустойчивостью алгоритма определе- ния локальных сдвигов и существенно затрудняющие удаление артефактов вызванных эффектом построчного переноса.

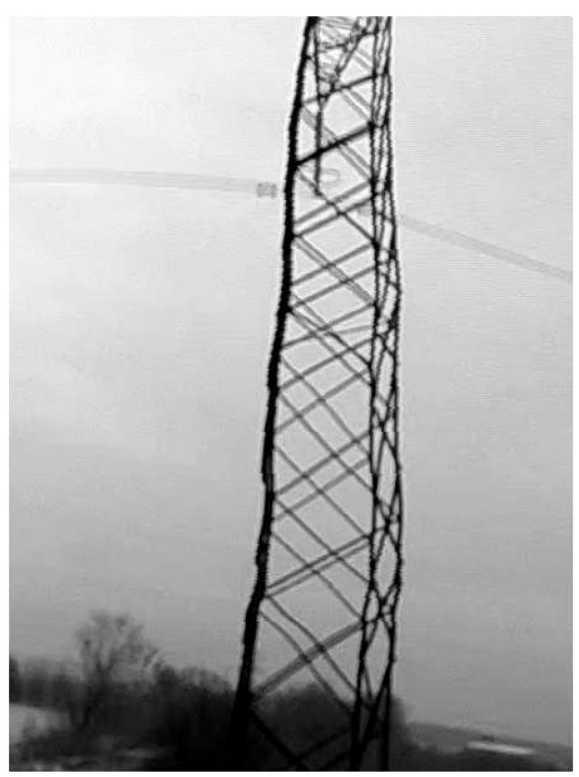

Рис. 3. Результаты обработки изображения опоры ЛЭП методом взвешенного оценивания для $\mathrm{s}_{\max }=5$

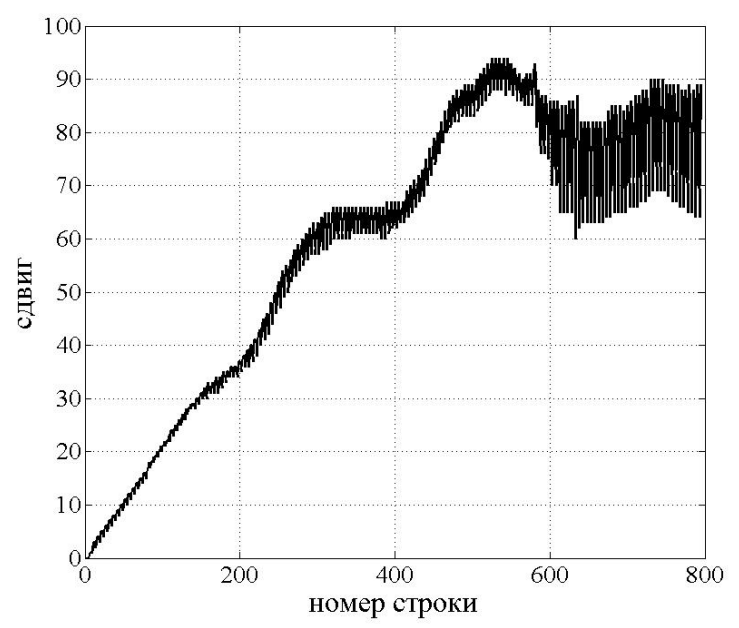

Рис. 4. График зависимости накопленного сдвига от номера строки для метода максимума ВКФ при $\Delta \mathrm{k}=5$

На рис. 5 показано негативное воздействие дрожаний, которое обуславливает размытие границ объекта. Для решения этой проблемы целесообразно применить к последовательности $s^{\prime}{ }_{k}$ медианную фильтрацию. Медианная фильтрация [9] - метод нелинейной фильтрации, эффективный при очистке сигналов от импульсных шумов, который не приводит к "размытию" сигнала. Из графика, приведенного на рис. 6, следует, что после медианной фильтрации последовательности $\mathrm{s}_{\mathrm{k}}$. $\mathrm{c}$ апертурой окна равной 5, дрожания практически полностью устраняются.

Эффективность данного метода проявляется при анализе обработанных изображений (рис. 7). Так, при выборе параметра $\Delta \mathrm{k}=5$ и обработке последовательности сдвигов медианным фильтром обработанное с апертурой окна равной 5 , изображение практически свободно от наклонов и искривлений, вызванных движением камеры. 


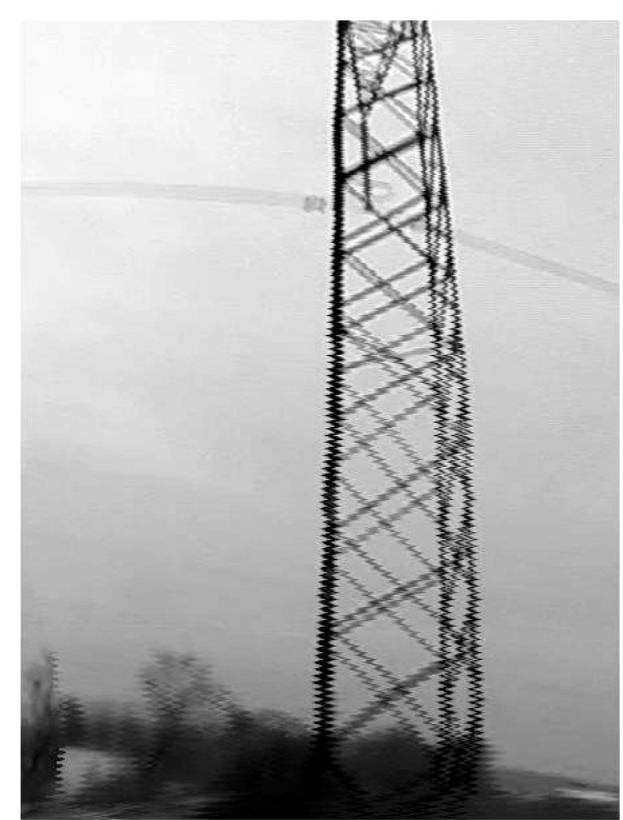

Рис. 5. Результаты обработки изображения опоры ЛЭП по методу максимума ВКФ при $\Delta \mathrm{k}=5$

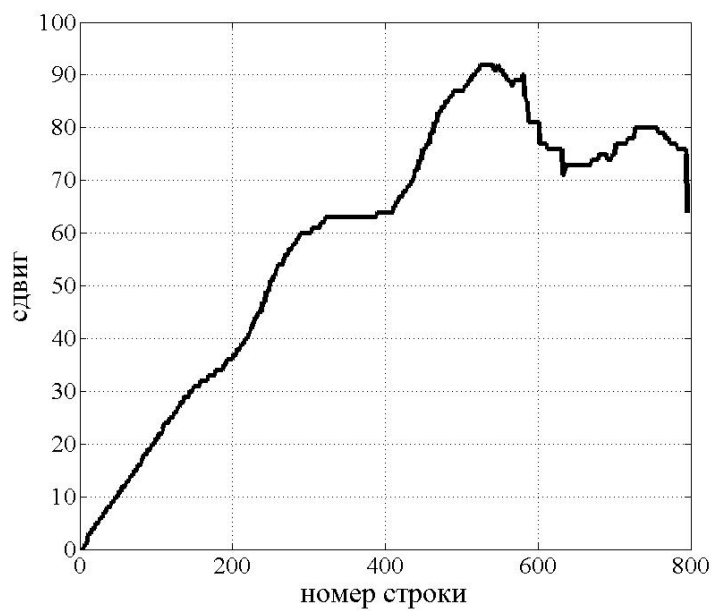

Рис. 6. График зависимости накопленного сдвига от номера строки для метода максимума ВКФ для $\Delta \mathrm{k}=5$, после обработки медианным фільтром

Дальнейшие исследования могут быть направлены на повышение устойчивости оценивания ВКФ, а также на выбор алгоритма обработки последовательности сдвигов $s_{k}^{\prime}$. Также может быть проведен спектральный анализ последовательности сдвигов с целью выделения частоты вибраций камеры и их последующей компенсации. Методы могут быть использованы для обработки термограмм.

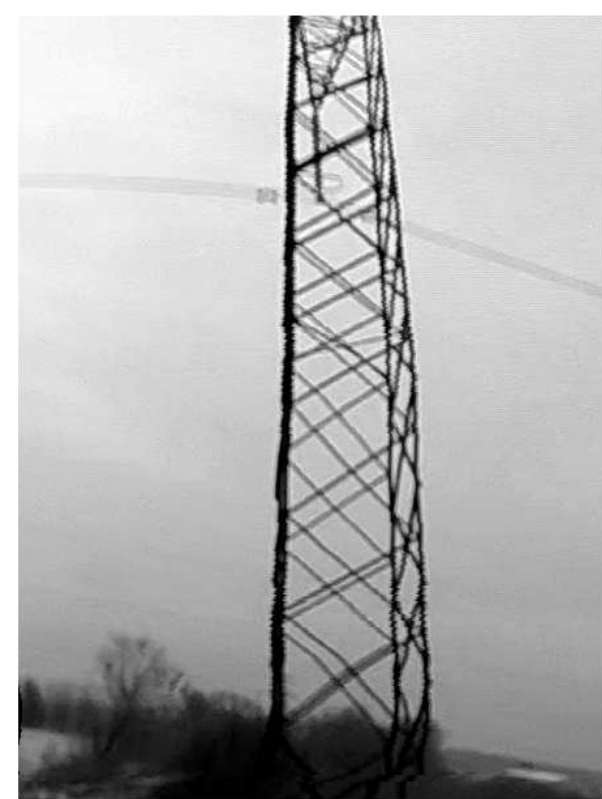

Рис. 7. Результаты обработки изображения опоры ЛЭП по методу максимума ВКФ для $\Delta \mathrm{k}=5$, после медианной фильтрации

\section{Выводы}

1. Обоснована необходимость удаления артефактов изображений, полученных в результате мониторинга энергетической инфраструктуры, связанных с построчным переносом.

2. Предложены методы, которые позволяют существенно снизить артефакты на изображениях, вызванные эффектом построчного переноса, что позволяет улучшить качество фото- и видеосъемки, производимой с БПЛА в процессе мониторинга.

3. Определен способ удаления размытых границ объектов вызванных дрожанием изображения при помощи медианной фильтрации.

4. Проведена разработка программного обеспечения и экспериментальные исследования реализации предложенных методов. Экспериментально показано, что методы позволяют практически полностью устранить артефакты на изображении, вызванные эффектом построчного переноса.

\section{СПИСОК ЛІТЕРАТУРИ}

1. Skarbek L., Zak A., Ambroziak D. Damage detection strategies in structural health monitoring of overhead power transmission system. -7 th European Workshop on Structural Health Monitoring. Clerk Maxwell, A Treatise on Electricity and Magnetism. 2014. 3rd ed., vol. 2. pp. 68-73. HAL Id : hal-01020412

2. Li L. The UAV intelligent inspection of transmission lines. - International Conference on Advances in Mechanical Engineering and Industrial Informatics. 2015. pp. 1542-1545. doi: 10.2991/ameii-15.2015.285

3. Adabo G. J. Unmanned aircraft system for high voltage power transmission lines of Brazilian electrical system. - Journal of Power and Energy Engineering. February 2014. 8(2). pp. 394-398. doi: 10.1049/oap-cired.2017.1048

4. Sheinin M., Schechner Y., Kutulakos N. Rolling shutter imaging on the electric grid. - IEEE International Conference on Computational Photography (ICCP). 2018. pp.1-12. doi: 10.1109/ICCPHOT.2018.8368472

5. Chun J.-B., Jung H., Kyung, C.-M. Suppressing rolling-shutter distortion of CMOS image sensors by motion vector detection. - IEEE Transactions on Consumer Electronics. 2008. Vol. 54, Is. 4. pp. 1479-1487. doi: 10.1109/TCE.2008.4711190

6. Baker S., Bennett E., Kang S., Szeliski, R. Removing rolling shutter wobble. - IEEE Computer Society Conference on Computer Vision and Pattern Recognition. 2010. pp. 2392-2399. doi: 10.1109/CVPR.2010.5539932 
7. Rengarajan V., Rajagopalan A., Aravind R., Seetharaman G. Image Registration and Change Detection under Rolling Shutter Motion Blur. - IEEE Transactions on Pattern Analysis and Machine Intelligence. 2017. Volume: 39, Issue: 10, pp. 19591972. doi: 10.1109/TPAMI.2016.2630687

8. Punnappurath A., Rengarajan V., Rajagopalan A. Rolling Shutter Super-Resolution. - IEEE International Conference on Computer Vision (ICCV). 2015. pp. 558-566. doi: 10.1109/ICCV.2015.71

9. Ali A., Rasha E., Alser T. Median Filter Performance Based on Different Window Sizes for Salt and Pepper Noise Removal in Gray and RGB Images. - International Journal of Signal Processing, Image Processing and Pattern Recognition. 2015. Vol.8, No.10, pp.343-352. doi: 10.14257/ijsip.2015.8.10.34

Рецензент: д-р техн. наук, проф. О. Г. Гриб, Національний технічний університет «ХПІ», Харків Received (Надійшла) 16.05.2018 Accepted for publication (Прийнята до друку) 10.08.2018

\section{Розробка методів видалення дефектів на зображеннях енергооб'єктів, отриманих в процесі дистанційного моніторингу за допомогою БПЛА}

\section{А. В. Івашко, А. О. Зуєв}

Метою статті є розробка та експериментальне дослідження методів видалення дефектів на зображеннях, отриманих в результаті дистанційного моніторингу об'єктів енергетичної інфраструктури за допомогою БПЛА, викликаного порядковим переносом зображення з матриці камери в пристрій зберігання. За час зйомки кадру об'єкт встигає змінити місце розташування, що обумовлено характером руху БПЛА, і в результаті ділянки одного кадру відображають різні моменти часу. Таке запізнення отримання даних з матриці щодо руху в кадрі призводить до появи добре помітних геометричних спотворень об'єктів. Камери з порядковим переносом, мають невисоку вартість, таким чином, їх застосування дозволяє істотно знизити вартість системи моніторингу, але вимагає розробки методів обробки отриманих зображень, які б мінімізували геометричні спотворення. При проведенні досліджень використовувалися методи кореляційного оцінювання відносного зсуву послідовностей що представляють собою рядки зображення, методи цифрової нелінійної фільтрації. Було проведено математичне моделювання в пакеті Scilab. Запропоновані і програмно реалізовані методи придушення ефекту порядкового перенесення, які не потребують аналізу послідовності кадрів $\mathrm{i}$ дозволяють усувати спотворення, викликані як нахилом відеокамери, так і її вібрацією. При цьому не потрібне проведення оцінки частоти коливань камери або виділення певних областей на зображенні. Використання запропонованих методів дозволяє створити програмне забезпечення для бортового комп'ютера БПЛА, яке може усувати артефакти на зображеннях, отриманих в процесі моніторингу в реальному масштабі часу. Що дозволяє спростити автоматичне виділення контурів і об'єктів на зображенні, а також визначення кількісних і якісних характеристик об'єктів за результатами фото- і відео-зйомки. Отримано розрахункові співвідношення, що дозволяють визначити передбачувані значення зсуву рядків зображення, викликаного ефектом порядкового перенесення. Крім зображень у видимому спектрі, запропоновані методи можуть бути використані для обробки термограм. Проаналізовано методи видалення спотворень на зображеннях та показано, що запропоновані методи дозволяють практично повністю усунути артефакти на зображенні, викликані ефектом порядкового перенесення.

Ключові слов а: повітряні лінії електропередачі, дистанційний моніторинг, БПЛА, порядкове перенесення, обробка зображень, медіанна фільтрація, взаємнокореляційна функція.

\section{Development of methods of removing defects in images of power facilities obtained in the process of remote monitoring using UAV}

\section{A. Ivashko, A. Zuev}

The purpose of the article is to develop and experimentally study methods of artifacts removal on images obtained as a result of remote monitoring of power facilities by UAVs caused by line-by-line transfer of an image from the camera to the storage device. During the capturing of a frame, the objects change position, which is due to the nature of the UAV's movement, and as a result, parts of a frame display different points in time. Such a delay in obtaining data from the camera relative to motion in the frame leads to the appearance of well-noticeable geometric distortions of objects. Cameras with line-by-line transfer are low cost, thus, their use significantly reduce the cost of the monitoring system, but requires methods for processing the obtained images that would minimize geometric distortions known as rolling shutter effect. During the research, methods of correlation estimation of the relative shift of sequences representing image lines, and digital nonlinear filtering were used. Mathematical modeling was carried out in the Scilab package. Methods for suppressing the rolling shutter effect, which do not require analysis of the sequence of frames, have been proposed and implemented in software. Thus, it makes possible to eliminate distortions caused by both the inclination of the video camera and its vibration. This does not require an assessment of the camera frequency or selection of certain areas in an image. Proposed methods can be implemented in for UAV on-board computer that can eliminate artifacts on images acquired during monitoring in real time. This enables to simplify the automatic selection of contours and objects on an image, as well as the estimation of quantitative and qualitative characteristics of objects based on the results of photo and video recording. Obtained the calculated relationships, which allow determining the expected values of the line shift of an image caused by the rolling shutter effect. In addition to images in the visible spectrum, the proposed methods can be used to process thermal images. The analyses show that the proposed methods can almost completely eliminate image artifacts caused by the rolling shutter effect.

Keywords: overhead power lines, remote monitoring, UAV, line transfer, image processing, median filtering, crosscorrelation function. 